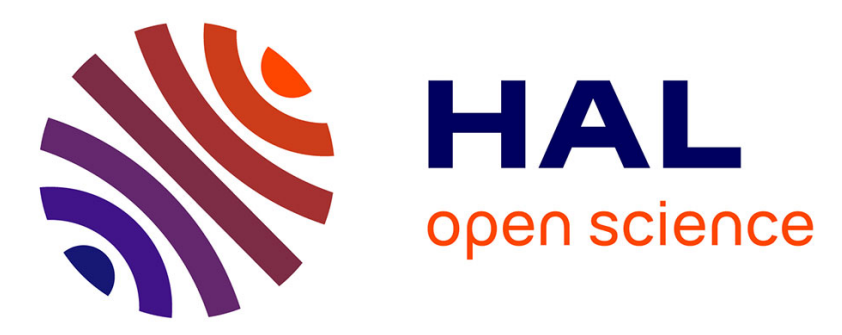

\title{
Low Noise CMOS Analog Front-End Circuit With an 8-bit 1-MS/s ADC for Silicon Sensors for Space Applications
}

Florent Bouyjou, Olivier Bernal, Hélène Tap, Jean-André Sauvaud, Pierre Jean

\section{To cite this version:}

Florent Bouyjou, Olivier Bernal, Hélène Tap, Jean-André Sauvaud, Pierre Jean. Low Noise CMOS Analog Front-End Circuit With an 8-bit 1-MS/s ADC for Silicon Sensors for Space Applications. IEEE Sensors Journal, 2014, 14 (5), pp.1617-1624. 10.1109/JSEN.2013.2295118 . hal-00981343

\section{HAL Id: hal-00981343 https://hal.science/hal-00981343}

Submitted on 22 Apr 2014

HAL is a multi-disciplinary open access archive for the deposit and dissemination of scientific research documents, whether they are published or not. The documents may come from teaching and research institutions in France or abroad, or from public or private research centers.
L'archive ouverte pluridisciplinaire HAL, est destinée au dépôt et à la diffusion de documents scientifiques de niveau recherche, publiés ou non, émanant des établissements d'enseignement et de recherche français ou étrangers, des laboratoires publics ou privés. 


\title{
Low Noise CMOS Analog Front-End Circuit With an 8bit 1MS/s ADC for Silicon Sensors for Space Applications
}

\author{
Florent Bouyjou, Olivier D. Bernal, Member, IEEE, Hélène Tap, Jean-André Sauvaud, and Pierre Jean \\ http://dx.doi.org/10.1109/JSEN.2013.2295118 (C) 2014 IEEE
}

\begin{abstract}
A low power analog front-end circuit for silicon $(\mathrm{Si})$ detectors has been fabricated in $0.35 \mu \mathrm{m}$ CMOS technology. It has been designed to read out signals from large-capacitance Si detectors for incident electron energy ranging from $50 \mathrm{keV}$ to 725 $\mathrm{keV}$. In order to quantify electron energy, the front-end integrates a charge preamplifier, a pulse shaper, a peak detector and an event-driven analog-to-digital converter (ADC). The complete front end including the ADC dissipates $2.5 \mathrm{~mW}$ for a maximum electron detecting rate of $650 \mathrm{kHz}$. The charge-to-voltage gain is approximately $60 \mathrm{mV} / \mathrm{fC}$ for a charge range of $0.6 \mathrm{fF}$ to $32 \mathrm{fF}$. The measured equivalent noise charge (ENC) is $3119 \mathrm{e}^{-}$for a $40 \mathrm{pF}$ detector parasitic capacitance.
\end{abstract}

Index Terms

Si Detector, Charge preamplifier, electron energy measurement.

\section{INTRODUCTION}

$\mathbf{P}$ ARTICLE instruments incorporate sensors that are used to convert particle energy into quantifiable electrical charges. These sensors with their corresponding analog electronics circuits, also called Analog-Front-End (AFE), form detection channels called "sensor heads" [1]-[3]. The necessity to improve both spatial and spectral resolutions requires the design of multichannel integrated electronics. Thus, space-borne detectors with Application Specific Integrated Circuits (ASICs) should be developed. These integrated circuits allow not only to perfectly adapt the readout circuits to each sensor in order to optimize performances but also to benefit from the various advantages inherent to the use of CMOS technologies: reduced power consumption, smaller size, shorter transit time signals [4] and higher integration [5]. In this paper, the designed front-end circuits are more particularly optimized for a $\mathrm{Si}$ detector used to obtain the energy spectrum of incident electrons ranging from $50 \mathrm{keV}$ to $725 \mathrm{keV}$ with an energy resolution of $10 \mathrm{keV}$. More precisely, this ASIC has been developed to be associated with a detector of radiation belt electrons, outside the South Atlantic Anomaly, on a low altitude polar orbiting spacecraft. Radiation belt electron fluxes at $600 \mathrm{~km}$ altitude are weak and their measurement implies a large transmission factor. The size of the detector allows reaching a transmission factor of $4 \mathrm{~cm}^{2}$.sr., which also allows to measure Lightning induced Electron Precipitation (LEP). Further, the spectrometer includes a collimator, which provides the possibility to obtain a rough pitch-angle distribution of the electrons (three directions).

A specific design methodology should be employed in order to withstand constraints due to space applications. The radiation environment can especially damage electronic systems on spacecraft and orbital satellite [6]. Two major radiation effects should be mentioned: the total cumulative dose called Total Ionizing Dose (TID) which is related to interactions between the semiconductor and the trapped particles, and transient events called Single Event Effects (SEE) resulting from high energy particles and random occurrences [7], [8]. Nowadays, instead of using technologies dedicated to space (such as specific BiCMOS or SOI) in order to improve the radiation hardness of integrated circuits, it seems appropriate to use specific design techniques (radiation hardening by design (RHBD)) [7], [9] applied on standard CMOS technologies which are less expensive [10], provide higher performances and for which parasitic effects are studied and well known [11], [12]. The choice of the technology depends first on the duration of the mission as well as on the radiative environments which is related to the space probe orbit trajectory. To fulfill this condition, we rely on studies of CMOS technologies radiation hardness already made by the scientific community [9]. Here, the sensor should be placed in a low Earth orbit satellite. It implies that the systems should withstand a $20 \mathrm{krad}$ TID for a space mission duration of 2 years. A $0.35 \mu \mathrm{m}$ CMOS technology has thus been chosen since it can naturally withstand more than $50 \mathrm{krad}$ [9]. In particular, it was shown that for CMOS technologies with a thin oxide thickness, TID effects are greatly reduced by tunneling mechanism. Further, in one of our previous $0.35 \mu \mathrm{m}$ CMOS design, it was shown that TID up to $360 \mathrm{krad}$ can be tolerated [13]. Further, the High-Voltage process option (HV) has also been chosen so that guard rings could be extensively used to prevent as much as possible latchup event. In addition, digital noise 


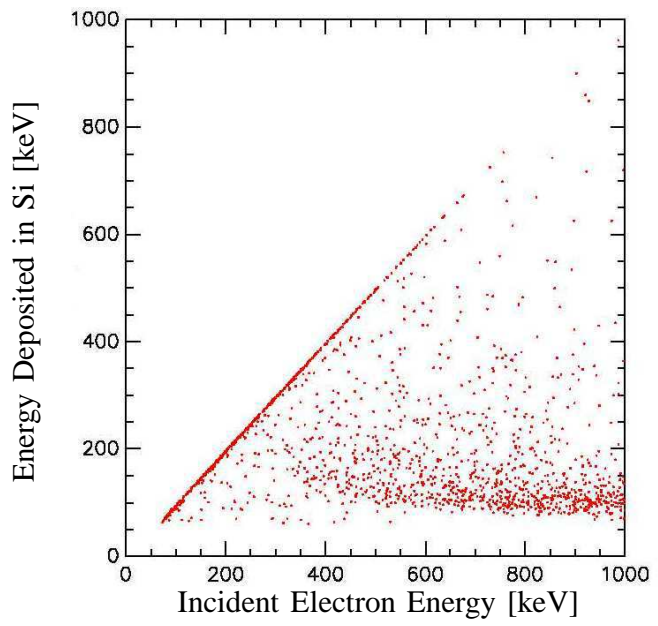

Fig. 1. GEANT4 Simulation of the energy deposited in a Si detector of $300 \mu \mathrm{m}$ thickness shielded by an aluminium foil of $6 \mu \mathrm{m}$ thickness versus the incident electron energy. $\mathrm{N}=200000$ incident electrons with a random incident angle $\theta \in\left[0 ; 10^{\circ}\right]$ and a random azimuth angle from $0^{\circ}$ to $360^{\circ}$.

that can affect the analog front-end in a mixed-signal circuit with both digital and analog circuits on a common substrate can be reduced by using triple well isolation available in $\mathrm{HV}$ technologies.

Here, in order to design and optimize the AFE circuits in terms of power consumption, linearity and noise, the main characteristics of the detector should be first extracted and modelled. Then, based on [14], some details about the frontend circuit design and its noise optimization are presented. Contrary to usual approches such as in [15], a differential input stage architecture has been preferred in order to be less prone to crosstalk issues between channels. Finally, simulation and measurement results are presented. A figure of merit (FoM) is also proposed to compare CMOS front-end circuits designed for various semiconductor detectors.

\section{Si CMOS FRONT END ARCHITECTURE}

Firstly, determining the model of the Si detector is necessary to design the Si detector circuits. As it will be explained in the following sections, the main parameters of such a model are the following ones: (1) the charge collection time $T_{c}$, (2) the input parasitic capacitance $C_{d e t}$ and (3) the leakage current $I_{0}$. After a short description of the Si detector model, these three parameters will thus be extracted.

\section{A. The detector model}

Here, the CMOS front-end is intented to be used with an electron energy spectrometer based on Si sensors. This instrument is intended to analyze the atmosphere-ionosphere-magnetosphere interactions during Transient Luminous Events (TLE) that can occur during atmospheric storms, in order to understand the physical mechanisms responsible for vertical impulsive coupling between the atmosphere and the ionosphere. The final aim is to assess the impact of these phenomena on the earth environment [16].

The Si detector is divided into four cells $(10 \mathrm{~mm}$ x $10 \mathrm{~mm})$ of $0.3 \mathrm{~mm}$ thickness and an aluminium foil of $6 \mu \mathrm{m}$ thickness is used to shield it from incoming visible and ultra-violet light. Based on Geant4 (see [17]) simulation results, electrons with energy higher than $50 \mathrm{keV}$ (due to aluminium foil) and lower than $500 \mathrm{keV}$ can be detected with a relatively good linearity (see Fig 1). Within such an energy range, the average number of electron-hole pairs $n_{\text {pair }}$ generated in the Si detector can be calculated as:

$$
n_{\text {pair }}=\frac{E_{\text {in }}}{\varepsilon_{S i}}
$$

where $E_{\text {in }}$ is the energy of the incident electron in $\mathrm{eV}$ and $\varepsilon_{S i}$ the silicon ionization energy $\left(\varepsilon_{S i}=3.62 \mathrm{eV}\right)$.

For energy higher than $500 \mathrm{keV}$, electrons can go through the Si semiconductor without losing significant amount of energy and therefore another kind of semiconductor (such as CdTe) would be required to detect them. However, as shown in Fig. 11, electrons with energy higher than $500 \mathrm{keV}$, can still be detected but with a much reduced detection probability. Consequently, the electron energy detection range has been extended up to $725 \mathrm{keV}$.

Knowing the maximum charge collection time $T_{c}^{\max }$ is important as it defines the maximum operating frequency of the instruments. It can be expressed as:

$$
T_{c}^{\max }=\frac{d^{2}}{\mu_{h} \times V_{0}}
$$


TABLE I

CHARACTERISTICS OF THE SI DETECTOR FOR $V_{0}=40 \mathrm{~V}$

\begin{tabular}{ccc}
\hline Characteristics & Symbol & $\mathrm{Si}$ \\
\hline \hline size $(\mathrm{mm})$ & $L \times l \times d$ & $10 \times 10 \times 0.3$ \\
generated pairs & - & $13.9 \times 10^{3}-2.01 \times 10^{5}$ \\
Equivalent charge (fC) & $Q_{\text {in }}$ & $2.2-32$ \\
Collection time $(\mathrm{ns})$ & $T_{c}^{\max }$ & $\approx 50$ \\
Parasitic capacitance $(\mathrm{pF})$ & $C_{\text {det }}$ & 34.8 \\
Leakage current $(\mathrm{nA})$ & $I_{0}$ & 2 \\
\hline
\end{tabular}

where $d$ represents the distance between the electrodes (which is approximately the Si thickness), $\mu_{h}$ the mobility of holes $\left(450 \mathrm{~cm}^{2} \cdot V^{-1} \cdot \mathrm{s}^{-1}\right)$ and $V_{0}$ the voltage applied across the electrodes.

Furthermore, the parasitic capacitance of the semiconductors (SCs) detector should also be taken into account in its electrical model since both the AFE bandwidth and noise depend on its value as it will be shown later. The equivalent capacitance of the $\mathrm{SC}$ can then be expressed as:

$$
C_{d e t}=\varepsilon_{0} \varepsilon_{r} \frac{A}{d}
$$

where $A$ is the area of the electrodes, $d$ the distance between them, $\varepsilon_{r}=11.8$ the relative permittivity of the Si material and $\varepsilon_{0}=8.854 \times 10^{-12} \mathrm{~F} \cdot \mathrm{m}^{-1}$ the vacuum permittivity.

Finally, the leakage current $I_{0}$ should also be taken into account since it increases the AFE output noise, affects the AFE dynamic range and may also compromise the AFE normal operation. This current $I_{0}$ is dominated by thermally generated electron-hole pairs that are separated by the applied electrical field. The typical measured value of the Si-detector leakage current is $2 \mathrm{nA}$ at $280 \mathrm{~K}$ which will be the maximal operating sensor temperature. Therefore, to design the circuit, a $10 \mathrm{nA}$ current leakage has been chosen as a worst case.

Table \ summarizes the main characteristics of the Si detectors. Based on this analysis, Si cells can be electrically modeled by a pulse current source $I_{d e t}$ to simulate a charge injection in parallel with the equivalent capacitor $C_{d e t}$ of the cell and the leakage current source $I_{0}$.

Knowing such parameters, the analog front end (AFE) consisting of the charge preamplifier (CPA) and pulse shaper (PS) can be designed.

\section{B. The Analog front End Design}

The designed AFE should be able to quantify the detected charge in order to reconstruct the electron energy spectrum. Consequently, the AFE should have a linear output response to ease the mapping of the electron energy. The first step is to convert charge into a voltage using a charge preamplifier (CPA) [1], [15]. The CPA consists of a transconductance amplifier (OTA) with a feedback capacitor $C_{f}(\approx 200 \mathrm{fF})$ to perform the charge integration and a resistor feedback $R_{f}(\approx 1.2 \mathrm{M} \Omega)$ to discharge $C_{f}$ and to provide a DC path for the detector leakage current. Then, in order to improve the Signal-to-Noise Ratio (SNR), the CPA output voltage is filtered by a circuit called pulse shaper (PS). As in [14], a first order semi-Gaussian PS (RC-CR bandpass filter) is used as PS for its high-speed response and its active bandpass filtering characteristics. These two blocks are widely used for such detectors [1]-[3], [14], [18]. The CPA and PS are presented together with the Si detector model in Fig 3 where $C_{p}$ represents the parasitic capacitance from the chip-sensor connection, the chip input pads and the CPA input capacitance.

The PS dynamic output range is set to $\Delta V_{S}^{P S_{\max }}=1.65 \mathrm{~V}$ in order to fit the input dynamic range of the ADC. Therefore, as stated in Table I in order to be able to collect a charge up to $32 \mathrm{fC}$, the Si chain gain can be calculated as follows:

$$
G_{C P A-P S}=\frac{\Delta V_{S}^{P S_{\max }}}{Q_{\max }} \approx 51.6 \mathrm{mV} / \mathrm{fC}
$$

As previously mentioned, the maximum operating frequency $f_{o p-\max }$ corresponds to the minimal shaping time of the PS $t_{s_{\min }}$, which must be equal to the maximal collection time $T_{c_{\max }}$ (see eq2) of the charges generated by an incident electron going through the Si detector. $T_{c_{\max }}$ is equal to $50 \mathrm{~ns}$ for our $\mathrm{Si}$ detector. Finally, to calculate the relaxation time of the chain, the conversion time of the SAR ADC must be taken into account. This ADC architecture has been chosen for reasons of low power consumption, small size and simplicity [19]. An 8-bits SAR ADC is used in adequacy in our application accuracy requirements and operates at a sampling frequency $\left(f_{a d c}\right)$ of $1 \mathrm{MHz}$. So, the conversion time $t_{c o n v}$ set by the channel to discriminate is given by:

$$
t_{c o n v}=\frac{1}{f_{\text {op-max }}}+\frac{1}{f_{a d c}}+T_{\text {reset }}
$$

with $T_{\text {reset }}$ the time required $(\approx 200 \mathrm{~ns})$ to reset the digital functions after the SAR ADC conversion. Note that $t_{\text {conv }}$ does not take into account the delays through the other Si chain blocks as they are negligible compared to the shaping and conversion times. 


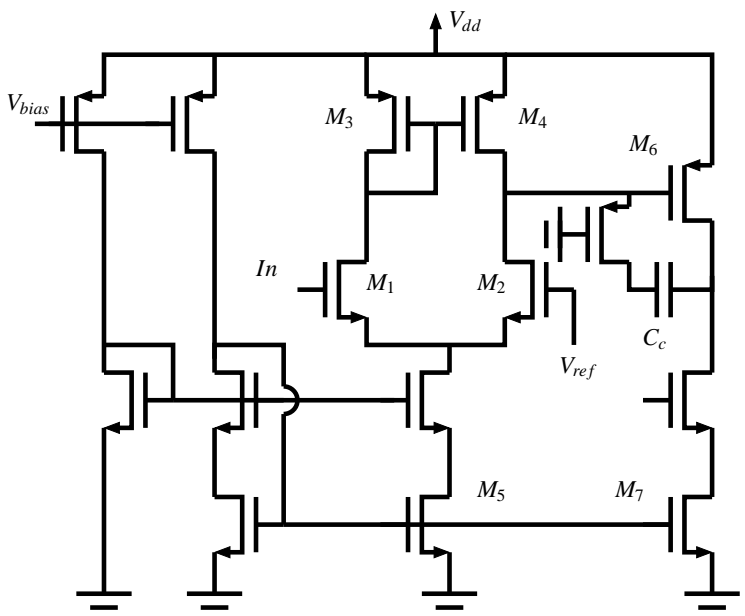

Fig. 2. Schematic of the operational transconductance amplifier (OTA) used as the charge preamplifier circuit

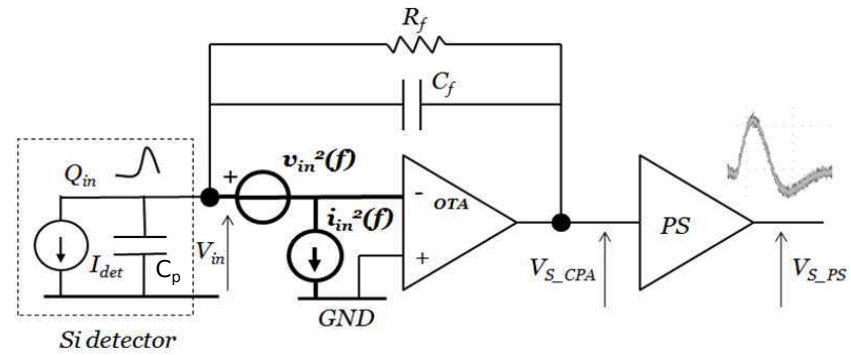

Fig. 3. Simplified AFE circuit diagram with Si detector model for noise analysis.

Furthermore, the maximum integration time is defined as the discharge time of the CPA+PS which corresponds to approximately 5 times the shaping time of the CPA+PS. This time must be inferior to the ADC conversion rate according to:

$$
t_{s_{\max }}=\frac{1}{5 \times f_{a d c}}=200 \mathrm{~ns}
$$

So, $t_{s}$ can be increased from $50 \mathrm{~ns}$ up to $200 \mathrm{~ns}$, which can be interesting to further reduce the circuit bandwidth and thereby the noise. However, $t_{s}$ is set to be equal to $100 \mathrm{~ns}$ in order to add a safety margin because the reset time of the chain (ADC reset and discharge time of the peak detector capacitance) must be taken into account.

As far as noise is concerned, the AFE has been designed to reduce both the intrinsic and extrinsic noise. With the aim to use up to 4 channels on the same die, crosstalk between channels must be drastically reduced. This issue is of up most importance here as each channel includes a peak-detector and a SAR ADC. Therefore, to address this extrinsic noise, a differential input stage (Fig 2) is used instead of the usual single-ended architecture [14], [15]. Nevertheless, the equivalent input noise power spectrum is doubled compared to a single transistor amplification stage (if each transistor is biased by the same current), which corresponds to a noise increase by a factor of $\sqrt{2}$.

For the intrinsic noise, studies show that most of the noise is generated by the CPA input transistor [18]. This implies that it should be designed properly. Fig 3 shows the circuit diagram that can be used to determine the output noise voltage of the AFE. The Equivalent Noise Charge (ENC) defined as the ratio of the total output rms noise of the PS to the peak amplitude response to one electron input charge, can be optimized either to the detriment of the power consumption by increasing the drain current $I_{D}$ or to the detriment of the bandwidth by increasing $t_{s}$ [18]. Here, the PS is limited to a first order band pass filter, in order to reduce the power consumption and circuit complexity. Based on [14], the ENC of the system including the Si sensor, the CPA and the PS $\mathrm{ENC}_{t o t}$ can be expressed as:

$$
\begin{gathered}
E N C_{t o t}=\frac{e}{q} \sqrt{R^{2}\left(\frac{2}{3} \frac{k T}{g m_{1} t_{s}}+\frac{K_{f}}{C_{o x}^{2}(W L)_{1}}\right)+\frac{q I_{t} t_{s}}{16}} \\
\text { with } R=C_{d e t}+C_{p}+C_{g s_{1}}+C_{g d_{1}}+C_{f}
\end{gathered}
$$

where $\mathrm{k}$ is the Boltzmann constant, q the elementary charge, $\mathrm{T}$ the temperature, $K_{f}$ the $1 / \mathrm{f}$ flicker noise coefficient, $I_{t}$ the sum of the detector leakage current and the equivalent input noise current of the input connection, $g m_{1}$ the $\mathrm{M}_{1}$ transconductance, 


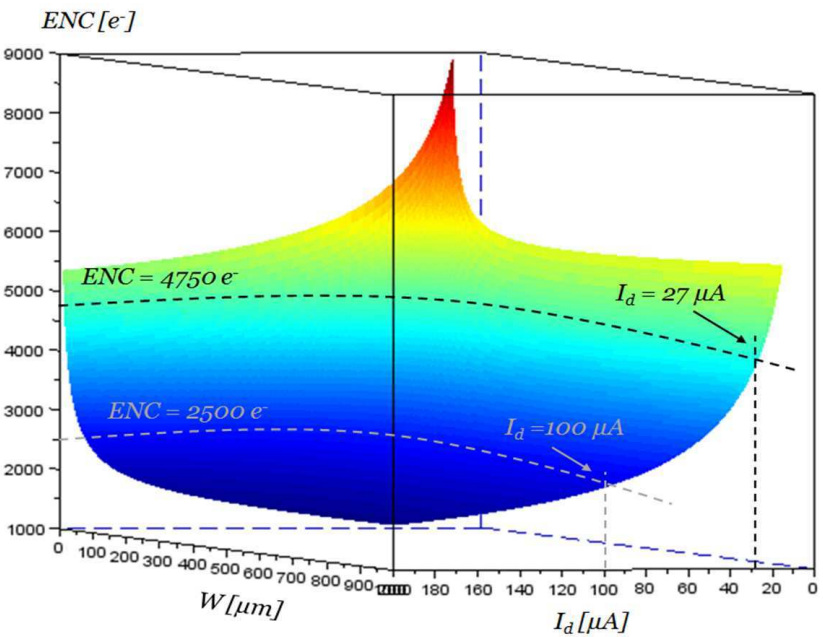

Fig. 4. 3D simulation of the CPA+PS circuit $\mathrm{ENC}_{\text {tot }}$ as a function of the width (W) and the drain current $I_{D}$ of the CPA input transistor with a first order band-pass filter, $C_{d e t}=40 \mathrm{pF}, C_{f}=200 \mathrm{fF}, t_{s}=100 \mathrm{~ns}, I_{0}=10 \mathrm{nA}$ and L=0.7 $\mu \mathrm{m}$.

$C_{g S_{1}}$ and $C_{g d_{1}} \mathrm{M}_{1}$ related capacitance and $C_{o x}$ the gate oxide capacitance per unit area.

A two-stage OTA is used for the CPA with an NMOS input transistor (Fig. 2). The design methodology is based on the separate study of the gain of each stage depending on the requested gain-bandwidth product which is affected by the detector capacitor, the feedback capacitor and the load capacitor. In addition, the transistors are biased in the weak or moderate inversion regions as such a biasing provides relevant advantages for high-gain/low-frequency applications compared to the strong inversion region [20]. However, one of the main drawback is that the slew rate decreases slightly in these regions [21]. Considering the design of the CPA, the drain current $I_{D}$ of the input transistor affects both the CPA noise and GBW. Therefore, out of these two CPA parameters, one should determine which one requires the higher current value. In Fig 4 and according to [2], the 3D simulation of the of the CPA+PS circuit in weak inversion as a function of the width (W) and the drain current $I_{D}$ of the input transistor is calculated with a first order bandpass filter and $t_{s}=100 \mathrm{~ns}$. Note that in Fig 4 , the capacitor $C_{d}$ is equal to the sum of the detector $C_{d e t}$ (included a variation of 10\%) and the parasitic input capacitor of about $1.5 \mathrm{pF}$ (pad and wire connections capacitors). A $27.5 \mu \mathrm{A} I_{D}$ is necessary to fulfill the minimum GBW requirement. However, as shown in Fig. 4 for such a current $I_{D}$, an $\mathrm{ENC}_{t o t}$ of $4750 \mathrm{e}^{-}$can only be reached. It is located in a steeply increasing $\mathrm{ENC}_{t o t}$ zone due to the thermal noise which is inversely proportional to the input transistor transconductance $g m_{1}$. It is thus preferable to increase the current up to $100 \mu \mathrm{A}$ to leave this zone and also to reduce the channel noise down to $2500 \mathrm{e}^{-}$ENC, even if it is to the detriment of power consumption. From these data, an electrical simulation with Cadence Spectre is performed to determine the required input transistor size to minimize the $\mathrm{ENC}_{t o t}: \mathrm{W}_{1}=556 \mu \mathrm{m}$.

\section{The Back End}

To save power as well as reduce the influence of any external parasitic signals, the analog to digital conversion of the CPA+PS output should be performed within the same chip. Thus, the following conversion channel architecture is opted: a CPA+PS, a comparator with an adjustable threshold voltage level, a peak detector (PD) and a 1 MS/s Successive Approximations Register (SAR) ADC (Fig 5). Such an SAR architecture has been chosen since it is a low power architecture that can easily achieve the required accuracy. Further, it only requires one active analog component: the comparator. To summarize, the CPA+PS converts the incident charge into a proportional voltage and the comparator detects if the incoming charge is higher or lower than the desired threshold level. Note that the minimum detection threshold level can be obtained by setting the threshold voltage of the comparator just above the noise floor. The PD is used to store the peak value of the PS output voltage, which is proportional to the electron energy. This track-and-hold voltage is then digitized by the SAR ADC. A control logic block of the system (referred as the "command block") is also designed to manage the communications between the blocks.

As it can be observed in Fig 6 six steps are necessary to convert the output signal of the PS:

1) Track the output voltage of the PS by the PD.

2) Detect the presence of an event: at the same time and independently of the PD actions, the comparator informs the Command block (EVENT $=$ ' 1 ') that an incoming charge has been detected. It is important to note that this signal is only sent when the comparator switches back to ' 0 ', which means when the amplitude of the peak falls below (after the PS peak) to be sure that the PD has finished the track-and-hold process.

3) Hold the charge value: The Command block sends (CMD_EN = '1') to the PD blocks which is blocked in order to store the value. Then, (CMD_READ = ' 1 ') is sent to the ADC to start the conversion (END_CONV = '0'). 


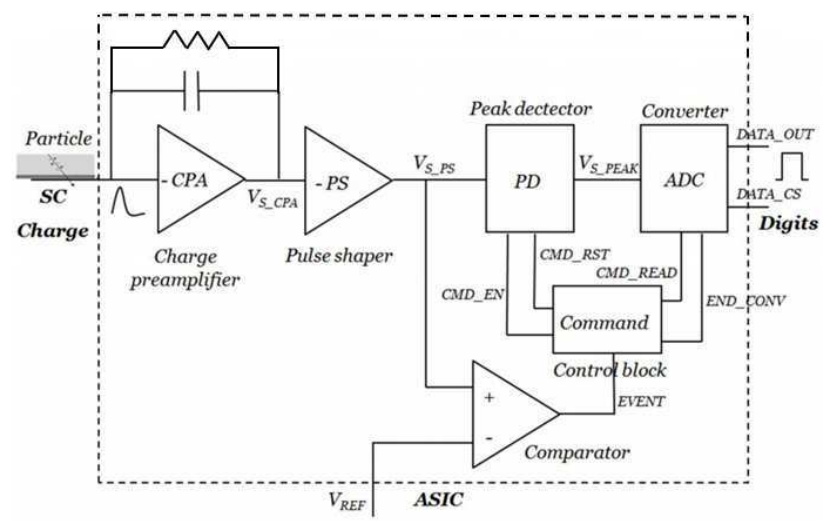

Fig. 5. AFE conversion system to measure the energy deposited by an incident electron in a Si detector.

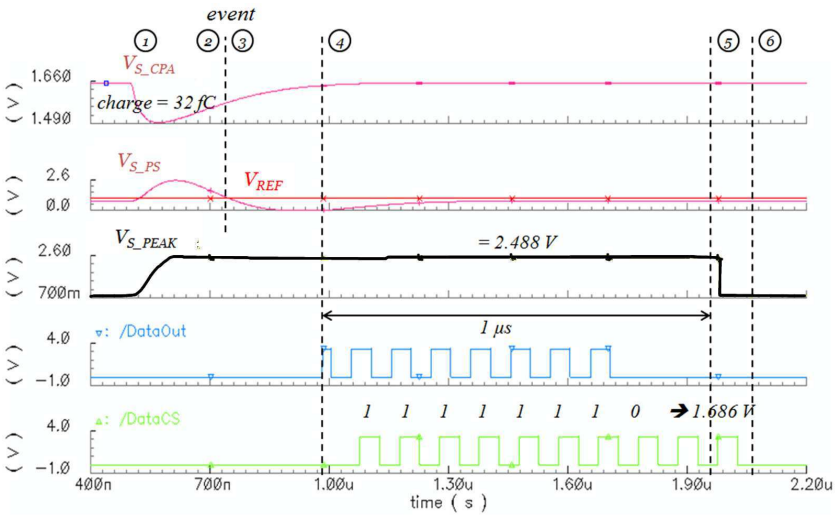

Fig. 6. From top to bottom: Transient output of the CPA, the PS, the PD and the ADC for a 50ns input charge of $32 \mathrm{fC}$ and $C_{d e t}=40 \mathrm{pF}$. The six steps necessary for the detection and the conversion can be observed.

4) Analog to digital conversion: The ADC converts the value while the others blocks of the back end are in sleep mode. When the conversion is complete, the ADC sends a signal (END_CONV =' 1 ') at the Command block and is set in standby mode.

5) The reset: the command unit resets the PD (CMD_RST = '1') in order to discharge the memory capacitor. Then CMD_READ is set to ' 0 ', which allows the ADC to recover its baseline.

6) Finally, the command system resets itself and sets $\left(\mathrm{CMD} \_\mathrm{EN}=\right.$ ' 0 ') and (CMD_RST $=$ ' 0 '). The system is then ready to perform a new conversion.

\section{RESULTS}

\section{A. Simulation}

Transient simulations are performed to observe the amplitude and the settling time corresponding to the charge conversion. The CPA and PS response times for charges from 1.6 to $32 \mathrm{fC}$ for a $50 \mathrm{~ns}$ injection duration $\left(T_{C_{\max }}\right)$, are respectively represented on the left and on the right of Fig77 A rise time of $23 \mathrm{~ns}$ and a fall time of $464 \mathrm{~ns}$ are obtained for the CPA. For the PS, a rise time of $67 \mathrm{~ns}$ and a fall time of $881 \mathrm{~ns}$ (including over-shoot) are obtained. The shaping time is found to be $t_{s}=110$ ns. The conversion range (from 1.6 to $32 \mathrm{fC}$ ) of the AFE is linear and shows a CPA resolution of $5 \mathrm{mV} / \mathrm{fC}$ and a CPA+PS resolution of $55 \mathrm{mV} / \mathrm{fC}$. The Si AFE circuits consume $1152 \mu \mathrm{W}$. The measured ENC is equal to $3277 \mathrm{e}^{-}$, which corresponds to a precision of $0.53 \mathrm{fC}$. These results are in agreement with the theoretical design methodology.

\section{B. Measurements}

The Si front end circuit has been implemented in a CMOS HV $0.35 \mu \mathrm{m}$ technology. Fig 8 shows the layout view of the complete front-end: CPA+PS, peak detector and ADC. The die size is about $0.21 \mathrm{~mm}^{2}$. To reduce crosstalk, analog and digital circuit wells are separated from each other and the corresponding circuits have their own power supplies VDD and GND. 

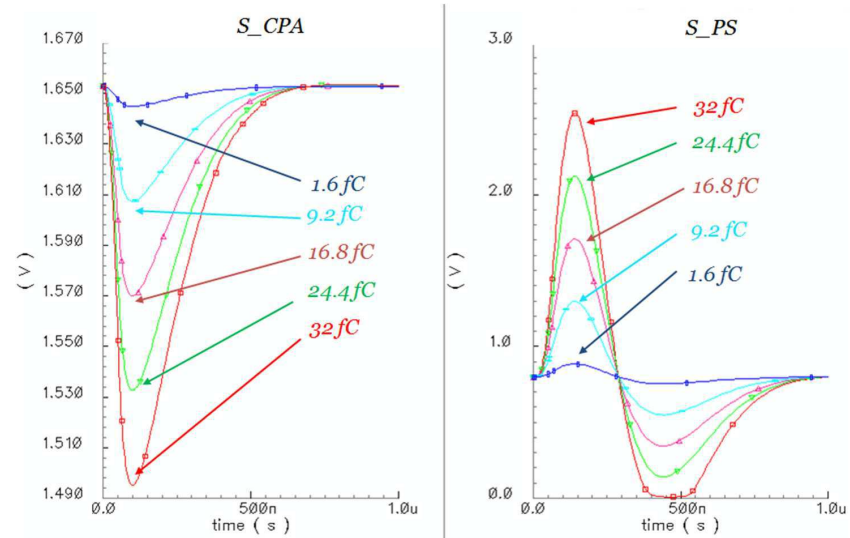

Fig. 7. Transient responses of the CPA output (left) and the CPA+PS output (right) for different injected charges ranging from $1.6 \mathrm{fC}$ to $32 \mathrm{fC}$, an injection duration of $50 \mathrm{~ns}$ and Cdet $=40 \mathrm{pF}$.

TABLE II

PERFORMANCES COMPARISON

\begin{tabular}{|c|c|c|c|c|}
\hline & This work & 【1] & {$[22]$} & 15 \\
\hline Year & 2013 & 2010 & 2011 & 2012 \\
\hline$\overline{\text { Detector type }}$ & $\overline{\mathrm{Si}}$ & $\overline{\mathrm{Si}-\mathrm{W}}$ & $\overline{\mathrm{Si}}$ & $\overline{\mathrm{CdZnTe}}$ \\
\hline Input charge range $(\mathrm{C})$ & $0.6 f-32 f$ & $0-2.4 p$ or $52 p$ & - & - \\
\hline Technology & AMS HV CMOS $0.35 \mu \mathrm{m}$ & AMS CMOS $0.35 \mu \mathrm{m}$ & UMC CMOS $0.18 \mu \mathrm{m}$ & Globalfoundries CMOS $0.35 \mu \mathrm{m}$ \\
\hline Detector capacitance $(\mathrm{pF})$ & 40 & $0-350$ & 30 & 6 \\
\hline Shaping time (ns) & 105 & 400 & 60 & 1000 \\
\hline Gain (mV/fC) & 51.5 & 55.5 & - & 100 \\
\hline $\mathrm{ADC}$ & yes & yes & yes & no \\
\hline Consumption (mW) & $<3$ & 2.8 & 1.75 & 7.8 \\
\hline $\operatorname{ENC}\left(\mathrm{e}^{-}\right)$ & 3119 & $2278+7.5 / \mathrm{pF}$ & 880 & 70 \\
\hline FoM & $\begin{array}{c}15 \times 10^{3} \\
3.4 \times 10^{4}(\mathrm{CPA}+\mathrm{PS})\end{array}$ & $\begin{array}{c}8.8 \times 10^{3}\left(C_{d e t}=40 \mathrm{pF}\right) \\
4.0 \times 10^{4}\left(C_{d e t}=350 \mathrm{pF}\right)\end{array}$ & $7.95 \times 10^{4}$ & $5.1 \times 10^{3}$ \\
\hline
\end{tabular}

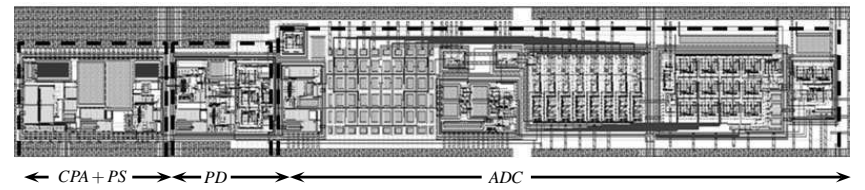

Fig. 8. Die layout of one Si CMOS front end channel implemented in a CMOS HV $0.35 \mu \mathrm{m}$ technology.

The linearity of the system can be evaluated by analyzing the ADC output. Its dynamic range is $44 \mathrm{~dB}$ with a linearity error of less than $0.32 \%$. After having characterized the behavior of the ADC, the output of the Si front end (DATA ${ }_{\text {OUT }}$ and DATA $\left._{C S}\right)$ are observed for a $50 \mathrm{~ns}\left(T_{C_{\max }}\right)$ input charge of $22.35 \mathrm{fC}$ (see Fig 9 ). For a charge of $22.35 \mathrm{fC}$, the digital output is '10101001'. The detection time corresponds to the time between the arrival of the charge and the start of its conversion at the output of the ADC $\left(\mathrm{DATA}_{C S}={ }^{\prime} 1\right.$ '). This detection time is equal to $320 \mathrm{~ns}$. The conversion time of the ADC is $1 \mu \mathrm{s}$ and the reset time of the digital blocks is $200 \mathrm{~ns}$. So, the effective operating frequency of the chains is approximately equal to 650 $\mathrm{kHz}$.

The linearity of the Si front end is plotted in Fig 9 for 50 acquisitions for each charge step and averaged over three different ASICs. The conversion gain is $57.7 \mathrm{mV} / \mathrm{fC}$ with an ENC of $3119 \mathrm{e}^{-}$(or $0.5 \mathrm{fC}$ ) in the linear region and the saturation value is approximately $32 \mathrm{fC}$ for linearity errors less than $0.81 \%$. The minimum detectable value is approximately $0.6 \mathrm{fC}$ which is higher than the expected detection threshold and is different from the measured ENC values. That can be explained by the fact that the detection thresholds are affected by the PCB ground noise.

Crosstalk measurements similar to those made in [13] have shown that crosstalk is negligible. So using an isolated-well technology (HV) and a differential pair in the CPA brings a real benefit in the integration of multiple channels on the same chip. In Table III, the specifications, the simulated and measured data are summarized. Measurements are very close to simulation results which validate our design methodology.

Certainly it is very difficult to compare the performance of a dedicated ASIC instrumentation to another because the detection and specifications are very different. For instance, the ASIC described in [1] was designed to be adapted to a wide range of detectors $\left(0<C_{d e t}<350 \mathrm{pF}\right)$ and to a wide range of charge detection $\left(0<Q_{i n}<52 \mathrm{pC}\right)$. That adaptability was made to the 


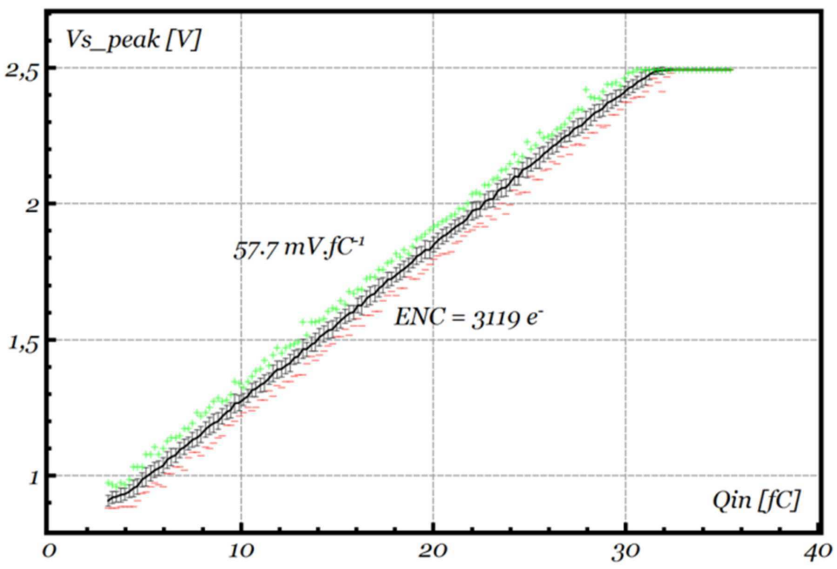

Fig. 9. Output Linearity of the Si CMOS front-end deduced from the output of the 8-bit ADC for injected charges ranging from $0.6 \mathrm{fC}$ to $35 \mathrm{fC}$ (50 acquisitions for each injected charge value) measured on 3 different ASICs with a $39 \mathrm{pF}$ input parasitic capacitance. Averages (line), standard deviations (error bars), maximum (green + ) and minimum (red -) values of 50 measures per step

TABLE III

Si CMOS FRONT-END PERFoRMANCE SUMMARY

\begin{tabular}{cccc}
\hline & Specification & Simulated & Measured \\
\hline \hline Input charge range $(\mathrm{fC})$ & $2.2-32$ & $0.53-32$ & $0.6-32$ \\
Detector capacitance $(\mathrm{pF})$ & 40 & 40 & $\approx 40$ \\
Shaping time $(\mathrm{ns})$ & 100 & 110 & $\approx 105$ \\
Gain $(\mathrm{mV} / \mathrm{fC})$ & 51.5 & 55.5 & 57.7 \\
Operating frequency $(\mathrm{kHz})$ & 1000 & 658 & 650 \\
Power supply voltage $(\mathrm{V})$ & 3.3 & 3.3 & 3.3 \\
Size $(\mu \mathrm{m})$ & & & $180 \times 1750$ \\
Consumption $(\mathrm{mW})$ & $<3$ & 2.5 & - \\
CPA+PS consumption $(\mathrm{mW})$ & & 1.15 & \\
PD consumption $(\mathrm{mW})$ & & 1.1 & \\
ADC consumption $(\mathrm{mW})$ & & 0.25 & \\
ENC $\left(\mathrm{e}^{-}\right)$ & 2500 & 3277 & 3119 \\
\hline
\end{tabular}

detriment of the speed, the gain and especially the noise $\left(>2278 \mathrm{e}^{-}\right)$. A cyclic ADC is also integrated and allows to achieve a 12 bit resolution. Conversely, the work presented in [3] was designed to be adapted to a specific application and a specific detector by minimizing the noise $\left(198 \mathrm{e}^{-}\right)$but to the detriment of the speed and the power consumption. In the present work, noise and power consumption are optimized for each channel for an electron-hole pair generation rate closely determined by the Si detector.

In order to evaluate different detector front-end performances more quantitatively, based on eq 7 , we propose to use the following Figure of Merit (FoM):

$$
F o M=\frac{C_{d e t}}{\text { Power } \cdot E N C_{t o t} \cdot \sqrt{t_{s}}}
$$

As previously explained, both the shaping time $t_{s}$ and $C_{d e t}$ are closely related to the detector performances (Table I) and as shown in eq7 they affect directly the ENC. They can thus hinder the performances of the CMOS front-end from being correctly assessed. Further, $t_{s}$ also reflects the maximum operation frequency that can be achieved. However, the power consumption of such circuits is usually determined by the noise performances to be achieved and not by the bandwidth. Therefore, $\sqrt{t_{s}}$ was chosen instead of $t_{s}$. Comparison results with other recent similar works [1], [15], [22] are given in Table [I]

\section{CONCLUSiON}

In this paper, a low noise low power CMOS front-end for particles energy measurement in space environment has been implemented in CMOS $0.35 \mu \mathrm{m}$ HV technology. A methodology for the electronic dimensioning perfectly adapted to the detector and the targeted application has been developed. The circuit occupies a surface area of about $0.21 \mathrm{~mm}^{2}$. The proposed circuit conversion gain is $57.7 \mathrm{mV} / \mathrm{fC}$ for an input dynamic range of $0.6 \mathrm{fC}$ to $32 \mathrm{fC}$ and the $\mathrm{ENC}$ is $3119 \mathrm{e}^{-}$. The power consumption is $2.5 \mathrm{~mW}$ at a rate of $650 \mathrm{kHz}$.

In order to extend the linear range of detection up to $4 \mathrm{MeV}$, in the future, another detector based on CdTe will be used in combination with $\mathrm{Si}$ detectors to fit the energetic electron detector instrument (IDEE) requirements for the TARANIS space mission [23]. Using the approach employed for the Si detectors, a CMOS front-end circuit dedicated to CdTe detectors caracterized by high parasitic capacitance and a lower electron/hole mobility than in $\mathrm{Si}$, will be designed. 


\section{REFERENCES}

[1] V. Bonvicini, G. Orzan, G. Zampa, and N. Zampa, "A double-gain, large dynamic range front-end asic with a/d conversion for silicon detectors read-out," Nuclear Science, IEEE Transactions on, vol. 57, no. 5, pp. 2963-2970, 2010.

[2] J. Kaplon and W. Dabrowski, "Fast cmos binary front end for silicon strip detectors at lhc experiments," Nuclear Science, IEEE Transactions on, vol. 52, no. 6, pp. 2713-2720, 2005.

[3] T. Kishishita, G. Sato, H. Ikeda, T. Takahashi, T. Kiyuna, and Y. Mito, "Low-noise analog asic for silicon and cdte sensors," Nuclear Science, IEEE Transactions on, vol. 57, no. 5, pp. 2971-2977, 2010.

[4] D. Orton, "Small, smart, intelligent and interactive handheld devices," VLSI Design Held jointly with 5th International Conference on Embedded Systems and Design., 19th International Conference on., p. 1, 2006.

[5] H. Baltes, O. Brand, A. Hierlemann, D. Lange, and C. Hagleitner, "Cmos mems - present and future," in Micro Electro Mechanical Systems, 2002. The Fifteenth IEEE International Conference on, 2002, pp. 459-466.

[6] C. Leroy and P. Rancoita, "Principles of radiation interaction in matter and detection," Hackensack, N.J.: World Sci., 2004.

[7] R. Lacoe, "Improving integrated circuit performance through the application of hardness-by-design methodology," Nuclear Science, IEEE Transactions on, vol. 55, no. 4, pp. 1903-1925, 2008.

[8] J. Schwank, M. Shaneyfelt, D. Fleetwood, J. Felix, P. Dodd, P. Paillet, and V. Ferlet-Cavrois, "Radiation effects in mos oxides," Nuclear Science, IEEE Transactions on, vol. 55, no. 4, pp. 1833-1853, 2008.

[9] P. Dodd, M. Shaneyfelt, J. Schwank, and J. Felix, "Current and future challenges in radiation effects on cmos electronics," Nuclear Science, IEEE Transactions on, vol. 57, no. 4, pp. 1747-1763, 2010

[10] H. Qu and H. Xie, "Process development for cmos-mems sensors with robust electrically isolated bulk silicon microstructures," Microelectromechanical Systems, Journal of, vol. 16, no. 5, pp. 1152-1161, 2007

[11] F. Caignet, S. Bendhia, and E. Sicard, "The challenge of signal integrity in deep-submicrometer cmos technology," Proceedings of the IEEE, vol. 89, no. 4 , pp. 556-573, 2001

[12] B. Ando, S. Baglio, P. Nouet, and N. Savalli, "Characterization of parasitic behaviors in cmos microsensors," in Instrumentation and Measurement Technology Conference, 2001. IMTC 2001. Proceedings of the 18th IEEE, vol. 3, 2001, pp. 1459-1462 vol.3.

[13] F. Bouyjou, O. Bernal, H. Tap, G. Picaut, and J.-A. Sauvaud, "Qualifying a cmos instrumentation chain for charged particles detection in the space environment," Analogue and Mixed-Signal Integrated Circuits for Space Applications. ESTEC - Noordwijk - The Netherlands, , Vol. 4th International, vol. 4, 2012

[14] F. Bouyjou, O. Bernal, H. Tap-Beteille, and J. Sauvaud, "A low-power cmos instrumentation chain for microchannel plates in astrophysics," Sensors Journal, IEEE, vol. 11, no. 4, pp. 1040-1045, 2011.

[15] J. Luo, Z. Deng, G. Wang, C. Cheng, and Y. Liu, "Design of a low noise readout asic for cdznte detector," in Nuclear Science Symposium and Medical Imaging Conference (NSS/MIC), 2012 IEEE, 2012, pp. 4098-4101.

[16] F. Lefeuvre, E. Blanc, J.-L. Pinçon, R. R.-D. andDavid Lawrence, J.-A. Sauvaud, J.-L. Rauch, H. de Feraudy, and D. Lagoutte, "Taranis-a satellite project dedicated to the physics of tles and tgfs.” Space Science Reviews, vol. 137, no. 1-4, pp. 301-315, 2008.

[17] J. Allison, K. Amako, J. Apostolakis, H. Araujo, P. Dubois, M. Asai, G. Barrand, R. Capra, S. Chauvie, R. Chytracek, G. A. P. Cirrone, G. Cooperman, G. Cosmo, G. Cuttone, G. G. Daquino, M. Donszelmann, M. Dressel, G. Folger, F. Foppiano, J. Generowicz, V. Grichine, S. Guatelli, P. Gumplinger, A. Heikkinen, I. Hrivnacova, A. Howard, S. Incerti, V. Ivanchenko, T. Johnson, F. Jones, T. Koi, R. Kokoulin, M. Kossov, H. Kurashige, V. Lara, S. Larsson, F. Lei, O. Link, F. Longo, M. Maire, A. Mantero, B. Mascialino, I. McLaren, P. Lorenzo, K. Minamimoto, K. Murakami, P. Nieminen, L. Pandola, S. Parlati, L. Peralta, J. Perl, A. Pfeiffer, M. Pia, A. Ribon, P. Rodrigues, G. Russo, S. Sadilov, G. Santin, T. Sasaki, D. Smith, N. Starkov, S. Tanaka, E. Tcherniaev, B. Tome, A. Trindade, P. Truscott, L. Urban, M. Verderi, A. Walkden, J. P. Wellisch, D. Williams, D. Wright, and H. Yoshida, "Geant4 developments and applications," Nuclear Science, IEEE Transactions on, vol. 53, no. 1, pp. 270-278, 2006.

[18] W. Sansen and Z.-Y. Chang, "Limits of low noise performance of detector readout front ends in cmos technology," Circuits and Systems, IEEE Transactions on, vol. 37, no. 11, pp. 1375-1382, 1990.

[19] H. Spieler, "Semiconductor detector systems," Oxford University Press, 2005.

[20] C. Enz, F. Krummenacher, and E. Vittoz, "An analytical mos transistor model valid in all regions of operation and dedicated to low-voltage and low-current applications," Analog Integrated Circuits and Signal Processing, vol. 8, no. 1, pp. 83-114, 1995. [Online]. Available: http://dx.doi.org/10.1007/BF01239381

[21] D. Comer and D. Comer, "Using the weak inversion region to optimize input stage design of cmos op amps," Circuits and Systems II: Express Briefs, IEEE Transactions on, vol. 51, no. 1, pp. 8-14, 2004.

[22] R. Kleczek, P. Grybos, and P. Otfinowski, "The design of low power low noise high speed cmos readout front-end electronics for silicon strip detectors," in Mixed Design of Integrated Circuits and Systems (MIXDES), 2011 Proceedings of the 18th International Conference, 2011, pp. 374-378.

[23] J.-L. Pincon, E. Blanc, P.-L. Blelly, M. Parrot, J. L. Rauch, J.-A. Savaud, and E. Seran, "Taranis 2014; scientific payload and mission strategy," in General Assembly and Scientific Symposium, 2011 XXXth URSI, 2011, pp. 1-4. 